\title{
Fungal Identification Method Based on DNA Sequence Analysis: Reassessment of the Methods of the Pharmaceutical Society of Japan and the Japanese Pharmacopoeia
}

\author{
Takashi Sugita*,a and Akemi Nishikawa ${ }^{b}$ \\ ${ }^{a}$ Department of Microbiology and ${ }^{b}$ Department of Immunobiology, Meiji Pharmaceutical University, 2-522-1 Noshio, Kiyose, Tokyo \\ 204-8588, Japan
}

(Received July 14, 2003; Accepted July 19, 2003)

\begin{abstract}
Fungal identification methods based on DNA sequence analysis were recently published in Methods of Analysis in the Health Sciences (Eisei-shiken-ho in Japanese) issued by the Pharmaceutical Society of Japan (PSJ) and in the draft second supplement to the 14th edition of the Japanese Pharmacopoeia (JP). We carefully compared and assessed the effectiveness of the two methods of fungal identification. The PSJ method analyzes D1/D2 26S rDNA, while the JP method analyzes the internal transcribed spacer (ITS) 1 region. The former method is superior to the latter in that the identification criterion described in the PSJ method is rationally defined based on the species concept, whereas distinct species can be misidentified as being of the same species using the identification criterion described in the JP method; and there are more sequence data for the D1/D2 $26 \mathrm{~S} \mathrm{rDNA}$ regions in the DNA data libraries (DDBJ/GenBank/EMBL) than for ITS1 data. Based on our assessment, we conclude that the PSJ method is superior to the JP method for fungal identification.
\end{abstract}

Key words — fungi, identification, DNA sequence

\section{INTRODUCTION}

There are approximately 70000 to 80000 species of fungi. While filamentous fungi are identified using mainly morphological characteristics, yeasts are identified using biochemical characteristics, such as their ability to utilize carbon and nitrogen compounds. However, these methods of identification are often problematic as there can be different mor-

*To whom correspondence should be addressed: Department of Microbiology, Meiji Pharmaceutical University, 2-522-1 Noshio, Kiyose, Tokyo 204-8588, Japan. Tel. \& Fax: +81-42495-8762; E-mail: sugita@my-pharm.ac.jp pho/biotypes within a single species. They are also time-consuming, and require a great deal of skill. To resolve these problems, the Health Pharmaceutical Committee of the Pharmaceutical Society of Japan (PSJ) proposed a "Fungal identification method based on DNA sequence analysis," which was subsequently described in the Methods of Analysis in the Health Sciences (Eisei-shiken-ho in Japanese) issued by the PSJ in 2002. ${ }^{1)}$ In this method, a partial region of the 26S subunit of the fungal rRNA gene is sequenced and compared with known fungal DNA sequences. As DNA sequence analysis methods are objective, reproducible, and rapid means of identification, they have been widely used..$^{2-4)}$ Recently, the "Rapid identification of microorganisms based on a molecular biological method" was proposed in the draft of the second supplement to the 14th edition of the Japanese Pharmacopoeia (JP) ${ }^{5,6)}$ However, there are marked differences between the PSJ and JP methods (Table 1). In this study, we compared and assessed the effectiveness of the two methods of fungal identification.

\section{MATERIALS AND METHODS}

Nucleotide Sequence Similarity —- Sequence similarities were compared using nuclear DNA relatedness values taken from the literature for the internal transcribed spacer (ITS) 1 and D1/D2 26S rDNA sequences separately. ${ }^{7)}$ Sequence similarity was determined visually from pairwise alignments. The nucleotide sequences used in this study were obtained from the DNA Data Bank of Japan (DDBJ, http://www.ddbj.nig.ac.jp).

Accumulation of DNA Sequences in DNA Data Libraries — All 685 species listed in the 4th edition of The Yeasts, A Taxonomic Study ${ }^{8)}$ were 
Table 1. Major Differences between the PSJ and JP Methods

\begin{tabular}{cll}
\hline \hline \multicolumn{1}{c}{ Item } & \multicolumn{1}{c}{ PSJ method } & \multicolumn{1}{c}{ JP method } \\
\hline Gene or region analyzed & $\begin{array}{l}\text { Domains 1 and 2 of the 26S subunit (D1/D2 26S } \\
\text { rDNA) (see Fig. 1) }\end{array}$ & $\begin{array}{l}\text { Internal transcribed spacer 1 region (ITS1) (see } \\
\text { Fig. 1) }\end{array}$ \\
Identification criterion & $\begin{array}{l}\text { Conspecific strains have more than 99\% nu- } \\
\text { cleotide similarity in the D1/D2 26S rDNA se- } \\
\text { quence }\end{array}$ & $\begin{array}{l}\text { Conspecific strains have more than 90\% nu- } \\
\text { cleotide similarity in the ITS1 region }\end{array}$ \\
\hline
\end{tabular}

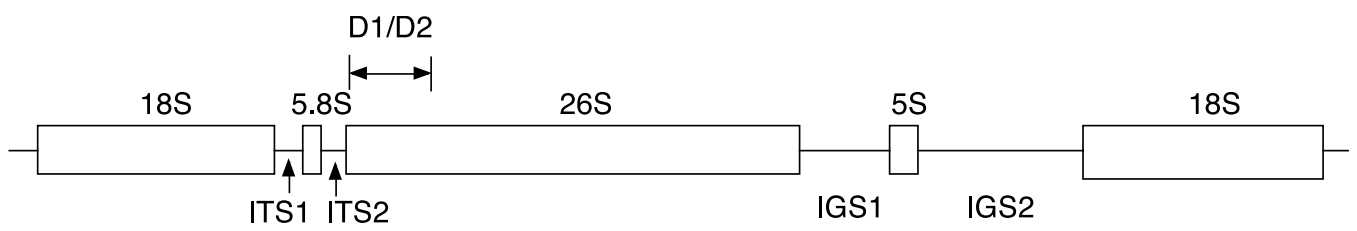

Fig. 1. Schematic Representation of the Fungal rRNA Gene

ITS, internal transcribed spacer; IGS, intergenic spacer; D1/D2, domains 1 and 2.

used in this study. The numbers of ITS1 and D1/D2 $26 \mathrm{~S}$ rDNA data that have been released from the DDBJ were examined. 18S rDNA sequences were also examined as a reference.

\section{RESULTS AND DISCUSSION}

The fungal rRNA gene consists of the 18S, ITS1, 5.8S, ITS2, 26S, intergenic spacer (IGS) $1,5 \mathrm{~S}$, and IGS2 regions (Fig. 1). The major differences between the PSJ and JP methods are summarized in Table 1.

\section{Identification Criteria}

There are approximately 70000 to 80000 species of fungi. Of these, the sequences of $5.8 \mathrm{~S}, 18 \mathrm{~S}$, D1/D2 26S rDNA, ITS, and IGS regions of almost all Trichosporon species have already been deposited in the DDBJ. As Trichosporon species are responsible for opportunistic infections, and are used as microbial biochemical oxygen demand (BOD) sensors, they are important yeasts from both the clinical and hygienic perspectives. In yeast taxonomy, a species concept has been defined using nuclear DNA relatedness values determined by a DNA-DNA hybridization experiment, which correspond well to biological relatedness. ${ }^{9)}$ Within a species, the DNA relatedness value is $70 \%$ or more (high relatedness group). Taxa with values of $40 \%$ to $70 \%$ are varieties of the same species or are sibling species (intermediate relatedness group). Between different species, the relatedness value is less than $40 \%$ (low re- latedness group). Figure 2 shows the relationships between the nuclear DNA relatedness value and ITS1 and D1/D2 26S rDNA sequence similarities for members of the genus Trichosporon. Figure 2 is based on the results for 34 pairs. In the high-relatedness group, the ITS1 and D1/D2 26S rDNA sequence similarities exceed 99\%. The JP method defines conspecific strains as having more than $90 \%$ similarity in the sequence of the ITS1 region, but there are distinct species that show more than $95 \%$ sequence similarity (Fig. 2). This suggests that comparing the DNA sequence similarity in the ITS1 region will lead to microorganisms in distinct species being misidentified as belonging to the same species. The identification criterion described in the JP method does not appear to be based on rational evidence, especially as regards the species concept. As far as we know, there have been no reports supporting the JP criterion.

In addition, analysis of D1/D2 26S rDNA sequences has the advantage that it not only enables species identification, but also permits phylogenetic analysis, unlike analysis of ITS1. Even when DNA sequence data from fungal isolates have not been registered in DNA data libraries and the isolate cannot be identified to the species level, the genus or family of the isolate can be identified from molecular phylogenetic analyses using D1/D2 26S rDNA sequences. In contrast, it is very difficult to deduce the phylogenetic position of a genus or family of an isolate from the ITS regions as they are more diverse than the $26 \mathrm{~S}$ region. 


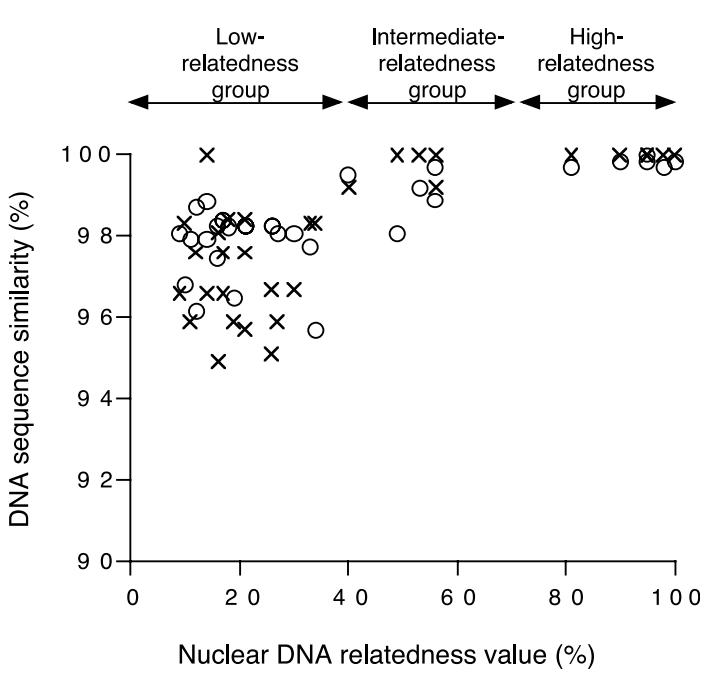

Fig. 2. Relationships between the Nuclear DNA Relatedness Value and the Similarities of the ITS1 and D1/D2 26S rDNA sequences

$\times$, ITS1; O, D1/D2 26S rDNA.

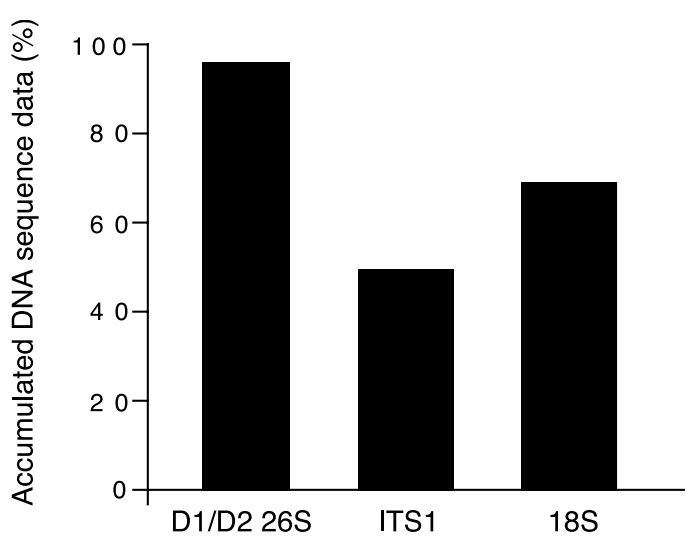

Fig. 3. Accumulated DNA Sequence Data in the DDBJ

\section{Accumulation of DNA Sequences in the DDBJ}

As there are an enormous number of filamentous fungi and their taxonomy is not well established, we focused on the sequence similarity in yeast species. Figure 3 shows accumulated DNA sequence data for ITS1, D1/D2 26S rDNA, and 18S rDNA in the DDBJ as of July 3, 2003. D1/D2 26S rDNA data of 666 species in a total of 685 yeast species identified have been released, while ITS1 data are available for only half of the species. Using $18 \mathrm{~S}$ rDNA sequences, a limited number of fungi species can be identified. However, 18S rDNA sequence analysis has not been widely used for fungal identification because approximately $1800 \mathrm{bp}$ must be determined for conclusive results. Nevertheless, $18 \mathrm{~S}$ rDNA data have been released for $70 \%$ of species. Since the
D1/D2 26S rDNA sequences of most yeast species have already been determined, the identification of the fungi using these sequences is practical.

In conclusion, the PSJ method is superior to the JP method as a standard fungal identification method.

\section{REFERENCES}

1) Health Pharmaceutical Committee (2002) (1.2.2.2.7) Fungal identification method based on DNA sequence. In Material of Public Health Council (Koushu-eisei-kyogikai-shiryo in Japanese), Pharmaceutical Society of Japan, Tokyo, pp. 5-6.

2) Fell, J. W., Boekhout, T., Fonseca, A., Scorzetti, G. and Statzell-Tallman, A. (2000) Biodiversity and systematics of basidiomycetous yeasts as determined by large-subunit rDNA D1/D2 domain sequence analysis. Int. J. Syst. Evol. Microbiol., 50, 13511371.

3) Kurtzman, C. P. and Robnett, C. J. (1997) Identification of clinically important ascomycetous yeasts based on nucleotide divergence in the $5^{\prime}$ end of the large-subunit (26S) ribosomal DNA gene. J. Clin. Microbiol., 35, 1216-1223.

4) Kurtzman, C. P. and Robnett, C. J. (1998) Identification and phylogeny of ascomycetous yeasts from analysis of nuclear large subunit (26S) ribosomal DNA partial sequences. Antonie Van Leeuwenhoek, 73, 331-371.

5) Ministry of Health, Labor and Welfare (2003) Drafts for the second supplement to JP 14. "Rapid identification of microorganisms based on molecular biological method." JP Forum, 12, 21-22 (Japanese), 35-37 (English).

6) Ministry of Health, Labor and Welfare (2003) Additional revision drafts for the second supplement to JP 14. "Rapid identification of microorganisms based on molecular biological method." JP Forum, 12,82 .

7) Sugita, T., Nishikawa, A. and Shinoda, T. (1994) Reclassification of Trichosporon cutaneum by DNA relatedness by using the spectrophotometric method and the chemiluminometric method. J. Gen. Appl. Microbiol., 40, 397-408.

8) Kurtzman, C. P. and Fell, J. W. (1998) Summary of species characteristics. In The Yeasts, A Taxonomic Study, 4th ed. (Kurtzman, C. P. and Fell, J. W., Eds.), Elsevier, Amsterdam, pp. 916-947.

9) Kurtzman, C. P. (1998) Nuclear DNA hybridization: Quantitation of close genetic relationship. In The Yeasts, A Taxonomic Study, 4th ed. (Kurtzman, C. P. and Fell, J. W., Eds.), Elsevier, Amsterdam, pp. 6368. 